\title{
Duration of Fever and Course of Symptoms in Young Febrile Children Presenting with Uncomplicated Illness
}

\author{
Marijke Kool, MSc, Gijs Elshout, MD, MSc, Henriette A. Moll, MD, PhD, \\ Bart W. Koes, PhD, Johannes C. van der Wouden, PhD, and \\ Marjolein Y. Berger, GP, PhD
}

Purpose: It is important to advise parents when to consult a doctor when their child has fever. To provide evidence-based, safety-net advice for young febrile children, we studied the risk of complications, the occurrence of alarm symptoms, the duration of fever.

Methods: In a 7-day prospective follow-up study, we included 463 consecutive children aged 3 months to 6 years who presented with fever at a general practitioner out-of-hours service. We excluded 43 children with complicated illnesses at presentation. In a structured assessment, the duration of fever before presentation was noted and a physical examination was performed. Parents reported alarming symptoms and rectal temperature in a diary for 1 week. The total duration of fever included its duration before presentation. Median duration of fever was estimated using the Kaplan-Meier test.

Results: During follow-up, 3.2\% of the children with uncomplicated illness at presentation developed a complicated illness. The presence of alarming symptoms dropped from $79.3 \%$ at day 2 of the fever episode to $36.7 \%$ at day 9 . The estimated median duration of the total fever episode was 4.0 days (95\% confidence interval, 3.6-4.4).

Conclusions: In children with uncomplicated illnesses, the daily occurrence of alarming symptoms reported by parents was high. The median duration of fever was 4 days. The predictive value of alarming symptoms reported by parents for complicated illness should be reconsidered. ( $\mathrm{J}$ Am Board Fam Med 2013;26:445-452.)

Keywords: Body Temperature, Child, Family Practice, Fever, Infant, Preschool Child

Feverish illnesses in children are common in primary care, but the prevalence of serious infections is low. ${ }^{1}$ Although most feverish illnesses (eg, respiratory infections) are self-limiting, fever is still a common reason to contact a general practitioner (GP) after office hours. ${ }^{2-5}$

Children with fever present in different stages of their illness, and alarming symptoms of serious

This article was externally peer reviewed.

Submitted 24 September 2012; revised 22 January 2013; accepted 4 February 2013.

From the Departments of General Practice (MK, GE, BWK, JCvdW, MYB) and General Pediatrics (HAM), Erasmus Medical Center, Rotterdam, the Netherlands; Sophia Children's Hospital, Rotterdam, the Netherlands (HAM); the Department of General Practice, EMGO Institute for Health and Care Research, VU University Medical Center, Amsterdam, the Netherlands (JCvdW); and the Department of General Practice, University Medical Center Groningen, University of Groningen, the Netherlands (MYB). disease may be absent at early presentation. Instructing parents of febrile children about what kind of alarming symptoms to expect during uncomplicated and complicated febrile illnesses, and when to contact the GP in the coming days of their child's illness, is important. This so-called safetynetting is a strategy to deal with situations of diagnostic uncertainty in otherwise uncomplicated illnesses. ${ }^{6}$ Building a safety net should include information on the existence of uncertainty, what

Funding: This study was supported by the Netherlands Organisation for Health Research and Development (ZonMw), dossier number: 42000012.

Conflict of interest: none declared.

Corresponding author: Marjolein Y. Berger, GP, PhD, Department of General Practice, University Medical Center Groningen, University of Groningen, PO Box 196, 9700 AD Groningen, The Netherlands (E-mail: m.y.berger@med. umcg.nl). 
to look for (ie, alarming symptoms), how exactly to seek further help, and what to expect regarding the time course. ${ }^{6}$ To give parents of young febrile children good safety-netting advice, it is essential to inform them about the risk of complications, the occurrence of alarming symptoms, the duration of fever, and the course of body temperature during follow-up.

To our knowledge, little is known about the course of uncomplicated feverish illnesses in primary care. Therefore, this study investigated the risk of complications, the presence of parent-reported alarming symptoms, the duration of fever, and the daily variation in body temperature among young febrile children with uncomplicated illnesses during a 1-week follow-up.

\section{Methods}

The study was performed at a GP out-of-hours service (OHS) in the southern part of Rotterdam, a large multiethnic city in the Netherlands. This GP OHS covers an area with approximately 300,000 inhabitants.

During midweek evenings and nights between December 13, 2004, and January 16, 2006, consecutive children aged 3 months to 6 years presenting with fever (as reported by the parents) were eligible for inclusion at the moment of presentation to the OHS. Fever had to be the main reason for encounter. Children were excluded if adequate communication in Dutch was impossible; if the child had already been enrolled in the study in the past 2 weeks; if there was no informed consent; or if they had a complicated illness at presentation (Figure 1).

A complicated illness was defined as a serious infection at presentation or admission to a hospital immediately after presentation. Serious infections included pneumonia, sepsis, meningitis, encephalitis, pyelonephritis, dehydration (caused by gastroenteritis or unknown cause), osteomyelitis, cellulitis, erysipelas, abscess, febrile convulsion, asthma exacerbation with fever, and, in children $\leq 1$ year old, bronchiolitis. Diagnosis of a serious infection was based on the diagnostic codes (registered according to the International Classification of Primary Care) noted by the GP in the OHS records. If a contact was not coded, a team of 3 GPs provided an International Classification of Primary Care code based on the (uncoded) diag-
Figure 1. Flow chart of children eligible for study analysis. GP, general practitioner.

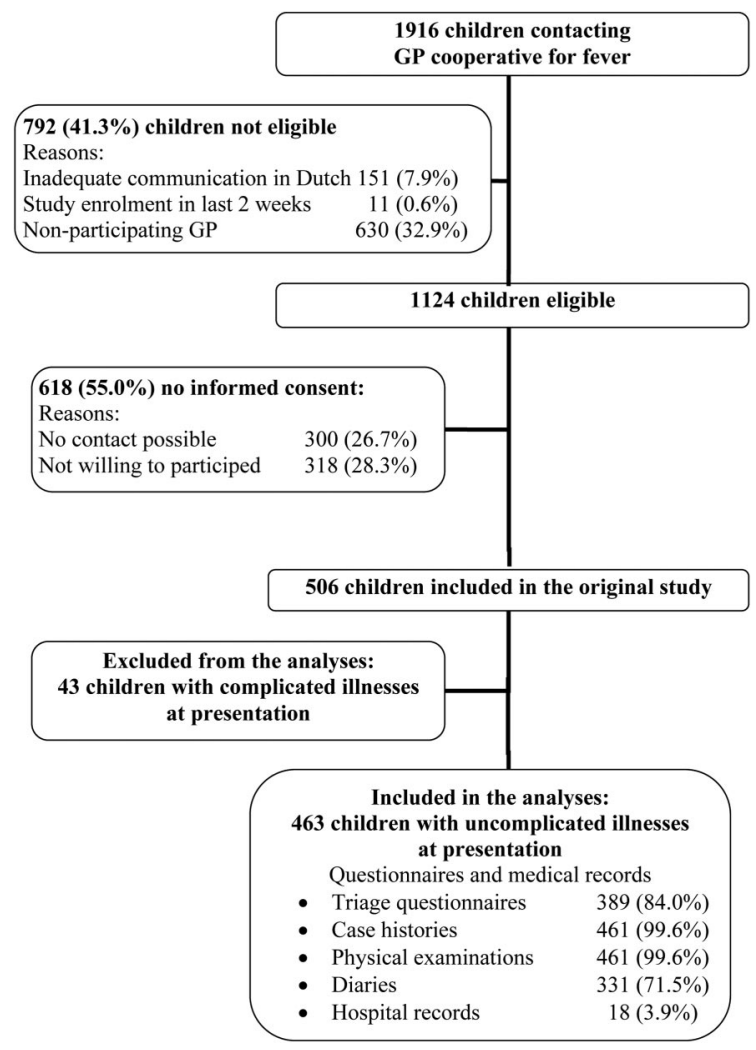

nosis made by the GP at the OHS or, if the diagnosis was missing, based on noted symptoms and findings during the history or physical examination. The team was blinded for triage result and the management by the GP at the OHS. Final coding was based on consensus. Children who were immediately referred at initial presentation were defined as having a serious infection if the pediatrician diagnosed the child as such. During follow-up, a complicated illness was defined as a serious infection diagnosed by a GP or a pediatrician or admission to hospital.

When parents phoned the OHS concerning their febrile child, the nurses performed their usual triage based on the triage guidelines of the Dutch College of General Practitioners ${ }^{7}$ and filled out a structured triage questionnaire in which parental concern (among other topics) was noted. According to these guidelines, a child was invited for consultation if the child was younger than 3 months of age, was very ill, was rapidly deteriorating, drank less than half of their normal consumption, had a rash that occurred during fever, was crying inconsolably, had a change in skin color, had a change in 
breathing pattern, was moaning or had apnea, had relevant comorbidity, had fever for $>3$ days or that was increasing after a fever-free period. In addition, a child was seen when the parents showed agitation, aggression, or persistent anxiety. A child was triaged as "self-care advice" if none of the above were present.

Based on this triage, the triage nurse gave advice over the telephone and arranged a face-to-face contact at the OHS or a home visit by a GP. GPs were free to prescribe treatments of their choice or to refer the patient.

An additional home visit by a trained research nurse was arranged as soon as possible after the evening or night of inclusion-but within 24 hours of inclusion-for all children included in this study. Using a structured questionnaire, the research nurse recorded demographic data, symptoms and signs at time of presentation, and medication use reported by the parents. In addition, the research nurse performed a structured physical examination, including rectal temperature. Parents received a thermometer and instructions to measure rectal temperature. During 1 week of follow-up, parents recorded in a structured diary rectal temperature twice a day and symptoms and medication use daily, starting at the day of the home visit. Diaries were returned by mail. If the parents did not return the diary, they were contacted by telephone within 1 month after the end of their follow-up and asked whether the child had had fever 1 week after the initial contact. Fever was defined as a temperature of $\geq 38.0^{\circ} \mathrm{C}$.

On the basis of national and international guidelines and before analyses, we defined 5 alarming symptoms. ${ }^{2,7}$ Drowsiness was defined as dull or difficult to awaken, no eye contact, or no or little reaction toward the parent. Inconsolable was defined as crying inconsolably, crying when picked up, very or very much irritated, or groaning. Abnormal circulation was defined as pale, ashen, or mottled skin. Dehydration was defined as drinking less than half that of normal or much less or no urine voiding. Shortness of breath was defined as rapid breathing.

Before the analyses we established cut offs for multiple choice questions. "No or little reaction toward the parent" was contrasted with "normal or almost normal reaction toward the parent." "Very or very much irritated" was contrasted with "not or slightly irritated." "Drinking less than half that of normal" was contrasted with "drinking half to twothirds that of normal" or "drinking two-thirds or more that of normal." "Much less or no urine voiding" was contrasted with "normal or slightly less than normal urine voiding." All other variables were dichotomous.

Antipyretic use before follow-up was defined as reported use of antipyretics at triage or reported use of antipyretics during this fever episode before presentation to the OHS. Daily antipyretic use during follow-up was defined as use of antipyretics reported in the diaries. Total antipyretic use was defined as antipyretic use before and during follow-up.

The Dutch Central Committee on Research Involving Human Subjects approved this study.

\section{Analyses}

Age is presented as median years and range. Temperature, duration of fever, and duration of antipyretic use are presented as mean \pm standard deviation, median and 5th (p5) and 95th (p95) percentiles. Data from children diagnosed with a serious infection or who were admitted to a hospital during follow-up were censored from all analyses from the day of diagnosis/admission onward.

Parents were asked about the first day of their child's fever as part of the structured assessment with the research nurse. This date was defined as the first day of the fever episode. In case of missing values $(n=2)$, the day of presentation was used as the first day of fever. The last day of fever was defined as the last known day of fever followed by $\geq 2$ days without fever reported in the diaries. In case of missing values in the diaries, the last day with fever reported (either in the diary or during the telephone call) was used as the last day of fever. The total duration of fever was defined as the period starting on the first day of fever and ending on the last day of fever in the diary. Duration of fever was measured in days. If a child was free of fever for $\geq 2$ days, a subsequent fever day was considered to be recurrent fever and not incorporated in the duration of the first fever episode. For the total duration of fever, the nonparametric $\mathrm{Ka}$ plan-Meier test was used; the survival function and the estimated median duration of fever with 95\% confidence intervals (CIs) are presented. Using the Kaplan-Meier test, a child was censored from the last known day of fever if it was 
unclear whether the child had had fever during the following 2 days. We performed this analysis for all children and for subgroups of children by age (3-11 months, $12-23$ months, and 24 months to 6 years).

To analyze differences between children presenting to the OHS on day 1 of their fever episode and children presenting after day 1 of their fever episode, comparisons between mean age, parental concern, reported temperature before presentation, and temperature during physical examination were performed using the Student $t$ test for continuous variables (age and temperature) or with the $\chi^{2}$ test for dichotomous variables (parental concern). Comparisons between height of temperature in the morning and the evening of the same day were performed using a paired Student $t$ test.

Statistical significance was set at $P<.05$. Analyses were performed with SPSS software version 17.0 for Windows (SPSS, Inc., Chicago, IL).

\section{Results}

Initially, 506 children were included in the study (Figure 1). Of these, 43 children (8.5\%) with complicated illnesses were excluded from the analyses because of either a diagnosis of serious infection at presentation $(\mathrm{n}=24,4.7 \%)$ or admission to a hospital directly after presentation $(\mathrm{n}=19,3.8 \%)$. A diagnosis at presentation was missing for 14 children included in the study. Finally, we analyzed

Table 1. Characteristics of Febrile Children With Uncomplicated illness $(n=463)$ Presenting to a General Practitioner (GP) Out-of-Hours Service

\begin{tabular}{|c|c|}
\hline Characteristics & $\begin{array}{l}\text { Children With } \\
\text { Uncomplicated Illness } \\
(\mathrm{n}=463)\end{array}$ \\
\hline Age in months, median (range) & $21(3-70)$ \\
\hline Male sex & $260(56.2)$ \\
\hline Immigrant & $235(50.8)$ \\
\hline \multicolumn{2}{|l|}{ Triage result } \\
\hline Face-to-face contact & $332(71.7)$ \\
\hline GP home visit & $0(0.0)$ \\
\hline $\begin{array}{l}\text { Duration of fever before presentation, } \\
\text { median days ( } 5 \text { th-95 th percentile) }\end{array}$ & $2.0(1.0-6.0)$ \\
\hline $\begin{array}{l}\text { Highest reported temperature before } \\
\text { presentation, median }{ }^{+} \mathrm{C} \text { ( } 5 \text { th- } \\
\text { 95 th percentile) }\end{array}$ & $39.5(38.1-40.6)$ \\
\hline $\begin{array}{l}\text { Use of antipyretics before start of } \\
\text { follow-up }\end{array}$ & $398(86.0)$ \\
\hline
\end{tabular}

Values are n (\%) unless otherwise indicated.

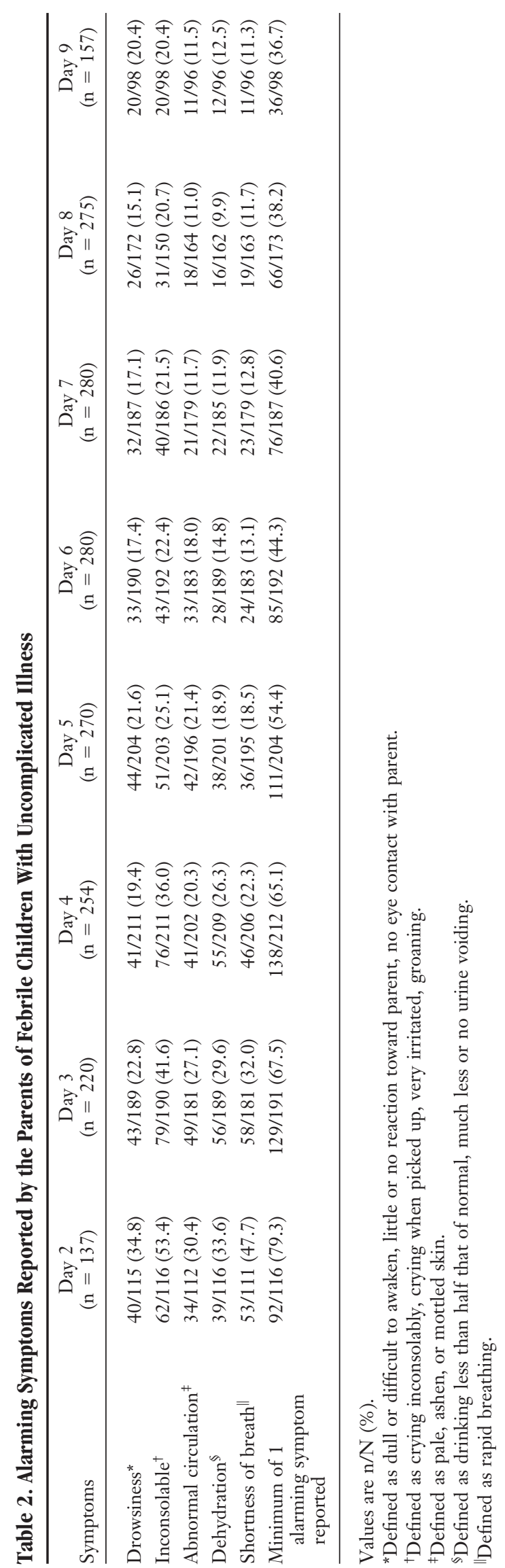


Figure 2. Rate of parental reported alarm-symptoms in febrile children with uncomplicated illnesses.

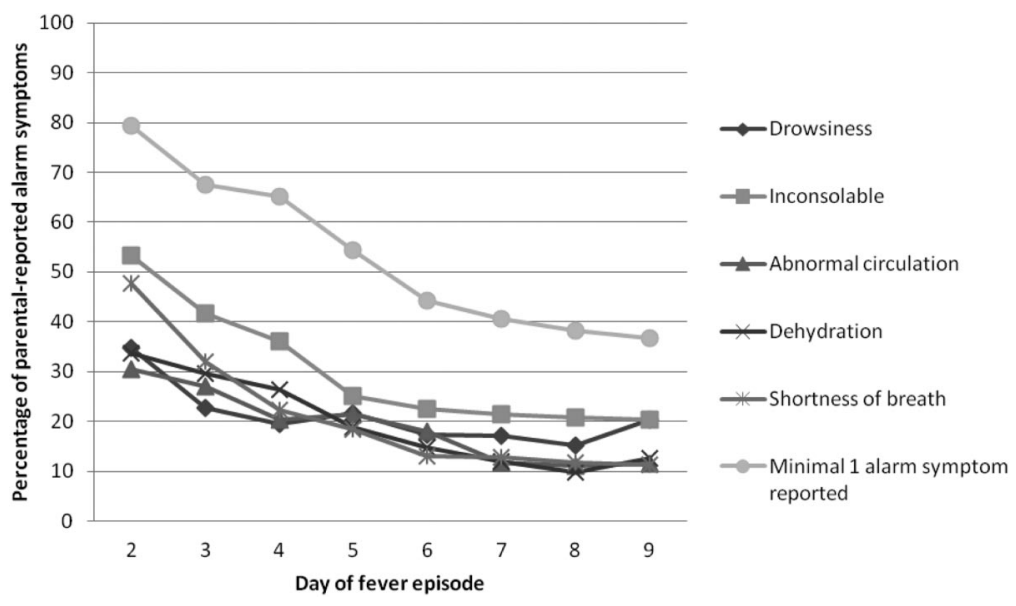

data of 463 children $(91.5 \%)$ with uncomplicated illness at presentation.

Diaries were returned for 331 children $(71.5 \%)$. Characteristics of the children are presented in Table 1 . The median highest reported temperature before presentation was $39.5^{\circ} \mathrm{C}(\mathrm{p} 5$, p95: $\left.38.1,40.6^{\circ} \mathrm{C}\right)$. Duration of fever before presentation was reported in 461 children (99.6\%). Median duration of fever before presentation was 2.0 days (p5, p 95: 1.0, 6.0 days). During followup, of the 463 children with uncomplicated illnesses at presentation, 15 (3.2\%) developed a complicated illness; they were diagnosed with a serious infection $(\mathrm{n}=4,0.9 \%)$ or admitted to hospital $(\mathrm{n}=11,2.8 \%)$. The median day on which a child developed a complicated illness was day 5 (p5, p95: day 2, day 11).

Of all 463 children, 206 (44.5\%) presented to the OHS on day 1 of their fever episode. These children did not differ from those who presented later during their fever episode with regard to age (mean age, 27 vs. 25 months; mean difference -2 months; $95 \%$ CI, -5 to 1 months) or degree of parental concern (worried or very worried vs. not worried or a little worried: $42.9 \%$ vs. $33.1 \% ; \chi^{2}=$ 3.312; df $=1 ; P=.07)$. However, these children did statistically significantly differ in highest reported temperature before presentation (39.4 vs. $39.6^{\circ} \mathrm{C}$; mean difference, $0.2^{\circ} \mathrm{C}$; $95 \% \mathrm{CI}, 0.0-0.3$ ) and in mean temperature at physical examination during the home visit $\left(37.8\right.$ vs. $37.5^{\circ} \mathrm{C}$; mean difference, $-0.4^{\circ} \mathrm{C} ; 95 \% \mathrm{CI},-0.5$ to -0.2$)$. Of 456 children, $189(41.4 \%)$ received antibiotic treatment during this fever episode.

\section{Alarming Symptoms}

Table 2 (Figure 2) presents the percentages of parent-reported alarming symptoms during the total fever episode. During follow-up, the presence of alarming symptoms decreased each day. The most frequent parent-reported alarming symptoms were inconsolable (decreasing from $53.4 \%$ at day 2 to $20.4 \%$ at day 9) and shortness of breath (decreasing from $47.7 \%$ at day 2 to $11.3 \%$ at day 9 ). At day 2, $79.3 \%$ of the parents reported at least one alarming symptom. At day 9 this percentage had decreased to $36.7 \%$ of the children with uncomplicated illness.

\section{Total Duration of Fever and Height of Fever}

The daily percentage of children with fever (temperature $\geq 38.0^{\circ} \mathrm{C}$ ) decreased from $65.0 \%$ at day 2 to $10.2 \%$ at day 8 of the total fever episode. The daily percentage of children with high fever (temperature $\geq 40.0^{\circ} \mathrm{C}$ ) decreased from $4.4 \%$ at day 2 to $0.0 \%$ at day 9 (Table 3). Figure 3 presents the course of fever in febrile children with uncomplicated illnesses. The estimated median total duration of fever was 4.0 days (95\% CI, 3.6-4.4). The estimated median total duration of fever was 4.0 days (95\% CI, 3.3-4.7) for children aged 3 to 11 months, 5.0 days (95\% CI, 4.1-5.9) for children aged 12 to 23 months, and 4.0 days $(95 \% \mathrm{CI}$, 3.4-4.6) for children aged 24 months to 6 years.

Of the 331 children for whom diaries were returned, fever recurred in $27(8.2 \%)$. The median day of recurrent fever was day 6 (p5, p95: day 3, day 24) after the start of the first fever episode.

Median morning temperature per day dropped from $37.9^{\circ} \mathrm{C}\left(\mathrm{p} 5, \mathrm{p} 95: 36.5,39.7^{\circ} \mathrm{C}\right)$ at day 2 to 
$37.0^{\circ} \mathrm{C}\left(\mathrm{p} 5, \mathrm{p} 95: 35.9,38.2^{\circ} \mathrm{C}\right)$ at day 9 of the total fever episode. Median evening temperature per day dropped from $38.1^{\circ} \mathrm{C}\left(\mathrm{p} 5, \mathrm{p} 95: 36.0,39.8^{\circ} \mathrm{C}\right)$ at day 2 to $37.2^{\circ} \mathrm{C}\left(\mathrm{p} 5, \mathrm{p} 95: 36.0,38.6^{\circ} \mathrm{C}\right)$ at day 9 of the total fever episode (Table 3). Temperature in the evening was significantly higher $\left(0.1-0.3^{\circ} \mathrm{C}\right) \mathrm{com}-$ pared with the morning temperature at days 3 to 6 and on days 8 and 9 of the total fever episode.

\section{Antipyretic Use}

Most children (398 of 459; 86.7\%) used antipyretics before follow-up (Table 1). During follow-up, daily use of antipyretics decreased from $40.2 \%$ at day 2 to $4.6 \%$ at day 9 . Of the 459 children, 411 $(89.5 \%)$ children used antipyretics during this fever episode.

\section{Discussion}

This study describes the risk of complications, the course of fever, and alarming symptoms during 7 days of follow-up in young febrile children with uncomplicated illnesses at presentation to OHS. In children with uncomplicated illness at presentation, $3.2 \%$ developed a complicated illness. Parent-reported alarming symptoms were frequent and decreased daily during follow-up. Estimated median duration of fever was 4.0 days (95\% CI, 3.6-4.4). On most days, temperature in the evening was only marginally higher than in the morning.

\section{Strengths and Limitations}

A strength of this study is that we prospectively collected daily alarming symptoms and daily body temperature in febrile children during 1 week after their presentation to OHS. This enabled us to describe the duration of fever and alarming symptoms in children with uncomplicated febrile illnesses at presentation. We present data for the total fever episode, not just starting from the presentation of the child to OHS. Children who presented at the first day of their fever episode did not differ from children who presented later.

A limitation of the study is that we had to collect data retrospectively for the days preceding the OHS contact. Although recall bias might have been present, the duration of fever before presentation had a median of only 2 days (p5, p95:1 day, 6 days); thus, based on this relatively short period of time, we assume that recall had no important effect on our analyses. 
Figure 3. Fever curve in young febrile children with uncomplicated illnesses $(n=463 ; 292$ events, 171 censored).
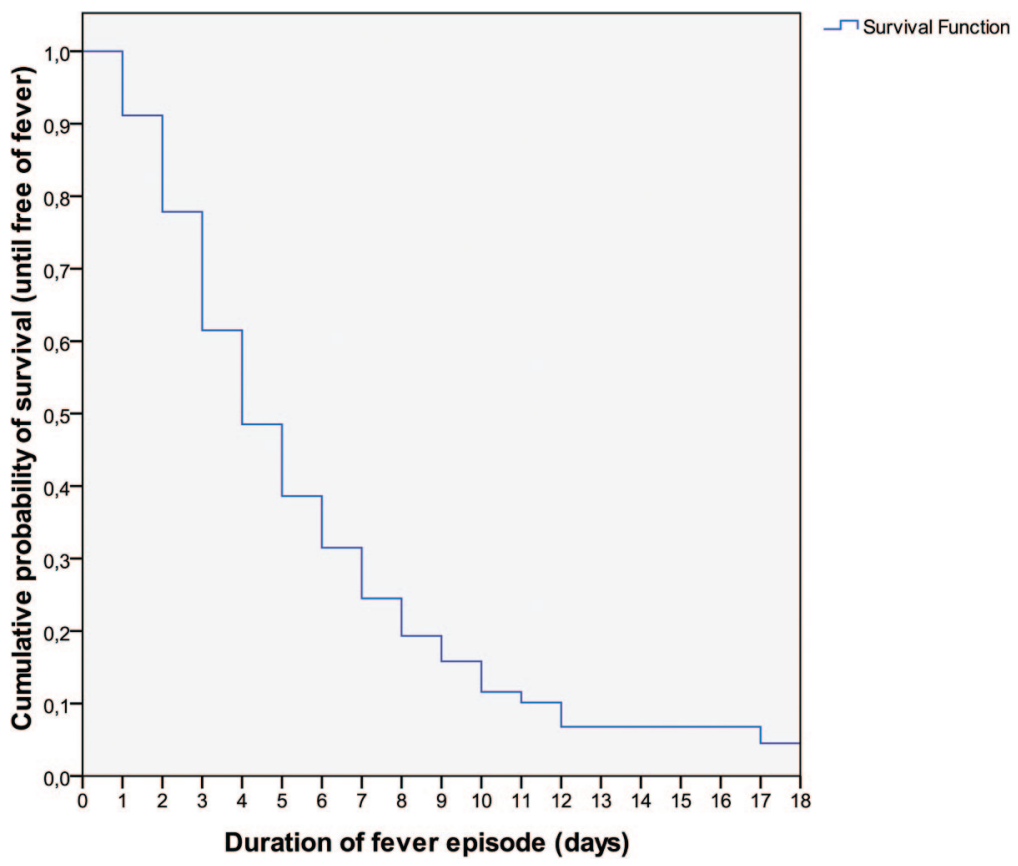

Alarming symptoms were frequently reported $(\leq 79.3 \%)$ in comparison to the relatively small percentage of serious illness (3.2\%). Many parents stopped filling out the diaries after their child recovered. Because the number of missing values in the diaries increased during follow-up, this might have resulted in an overestimation of the percentage of alarming symptoms at the end of follow-up. However, this cannot explain the discrepancy between the frequency of parent-reported alarming symptoms and the $3.2 \%$ of children who developed a complicated illness during the week of follow-up. This study indicates that parent-reported alarming symptoms as a trigger for reconsultation will give raise to a high percentage of false positive reconsultations. Parent-reported alarming symptoms may have been frequently reported because they also occur in children with uncomplicated illness or parents are not aware how to interpret the description of alarming symptoms given by their physician.

\section{Comparison With Existing Literature}

We found no other studies that describe the total duration of fever episodes in children in general practice. Maguire et $\mathrm{al}^{8}$ described a median duration of illness of 3 days, which was queried retrospectively in a study on how parents access acute services for febrile children; however, they did not prospectively follow the febrile children for the total duration of the fever episode. A systematic review found 7 studies of the predictive value of duration of fever for serious infections at the moment of presentation in 1644 febrile children; however, because of a lack of information on the duration of fever, no conclusions could be drawn about the predictive value for serious infection. ${ }^{9}$

\section{Implications for Future Research and Clinical Practice}

This study describes the risks of complicated illness, parent-reported alarming symptoms, and duration of fever in young febrile children with uncomplicated illnesses. The median duration of fever was 4 days, and parents frequently reported alarming symptoms in children with an uncomplicated illness. To prepare safety-netting advice about alarming symptoms during the fever episode in children with uncomplicated illnesses, parent-reported alarming symptoms need to be reconsidered and validated. The cost effectiveness of advising reconsultation in the presence of alarming symptoms might be low and needs further evaluation.

The authors thank all the parents and children who participated in this study. 


\section{References}

1. Van den Bruel A, Bartholomeeusen S, Aertgeerts B, Truyers C, Buntinx F. Serious infections in children: an incidence study in family practice. BMC Fam Pract 2006;7:23.

2. National Collaborating Centre for Women's and Children's Health. Feverish illness in children. In: Assessment and initial management in children younger than 5 years. 1st ed. London: RCOG Press; 2007:1-16.

3. Bruijnzeels MA, Foets M, van der Wouden JC, van den Heuvel WJA, Prins A. Everyday symptoms in childhood; occurrence and general practitioner consultation rates. Br J Gen Pract 1998;48:880-4.

4. Hay AD, Heron J, Ness A; ALSPAC study team. The prevalence of symptoms and consultations in pre-school children in the Avon Longitudinal Study of parents and children (ALSPAC): a prospective cohort study. BMC Fam Pract 2005;22:367-74.

5. Crocetti M, Moghbeli N, Serwint J. Fever phobia revisited: have parental misconceptions about fever changed in 20 years? Pediatrics 2001;107:1241-6.

6. Almond S, Mant D, Thompson M. Diagnostic safety-netting. Br J Gen Pract 2009;59:872.

7. Dutch College of General Practitioners. [Dutch College of GPs' Telephone guideline for triage and advice]. Utrecht: NHG; 2002.

8. Maguire S, Ranmal R, Komulainen S, et al. Which urgent care services do febrile children use and why? Arch Dis Child 2011;96:810-6.

9. Elshout G, Monteny M, van der Wouden JC, Koes BW, Berger MY. Duration of fever and serious bacterial infections in children: a systematic review. BMC Fam Pract 2011;12:33-9. 